\section{Notes}

1 For instance, while explaining how the emphasis on social harmony that is central to Karen ideology may assist in the maintenance of order within the refugee camp, the author fails to address in any meaningful way the common criticism that prioritizing community harmony in the administration of justice is often at the expense of individual rights and well-being (109).

2 See Barbara Harrell-Bond, "Can Humanitarian Work with Refugees be Humane?” Human Rights Quarterly 24 (2002): 51.
Anna Purkey is a member of the Quebec Bar Association and holds a BCL/LLB from McGill University, an LLM from University of Toronto, and is finishing her doctorate in civil law at McGill University. Her current research focuses on the legal empowerment of refugees in protracted refugee situations and includes a longstanding interest in the situation along the Thai-Burmese border. The author may be contacted at anna.purkey@mail.mcgill.ca.

\title{
The International Law of Migrant Smuggling
}

\author{
Anne T. Gallagher et Fiona David \\ Cambridge University Press, 2014: ISBN 987-1-107-01592-o (Hardback), 783 p.
}

$\mathrm{L}$ es migrations internationales ont été - et demeurent toujours - un élément constant et influent de l'histoire humaine. Elles ont soutenu le processus de croissance économique mondiale, contribué à l'évolution des États et des sociétés et enrichi de nombreuses cultures et civilisations. Les migrations constituent un laboratoire privilégié de l'évolution du droit international général depuis les origines de cette discipline. Force est cependant de constater que la dialectique entre migrations et droit international est encore très largement insuffisamment connue. Le fossé grandissant entre la réalité du mouvement migratoire dans un monde de plus en plus interconnecté et son encadrement normatif demeure, à n'en point douter, un enjeu contemporain majeur. Malgré les travaux et les efforts entrepris depuis plus d'une décennie au plan international et l'accroissement considérable des activités des groupes criminels organisés en matière de trafic illicite de migrants portant gravement préjudice aux États et mettant en danger la vie ou la sécurité des migrants concernés, il n’y a aucun instrument universel qui porte sur tous les aspects du trafic illicite de migrants.

Rattrapé par l'actualité médiatique dramatique, le droit est confronté à un constat synchronique amer, ses règles et principes répondent mal aux problèmes auxquels les États sont confrontés surtout en ce qui concerne le contrôle de l'immigration, la prévention des trafics et la traite des êtres humains. Le durcissement progressif et quasi généralisé des politiques migratoires causé par la multiplication de mesures contre l'immigration irrégulière, diminue d'autant les possibilités légales de migration, créant ainsi un environnement propice à l'augmentation du trafic de migrants. En droit international, le trafic illicite de migrants désigne «le fait d'assurer, afin d'en tirer, directement ou indirectement, un avantage financier ou un autre avantage matériel, l'entrée illégale dans un État Partie d'une personne qui n'est ni un ressortissant ni un résident permanent de cet État $»^{1}$.

Pour autant, les évènements tragiques de ces dernières années, aggravés par les crises et les conflits actuels de toutes sortes, ont permis une prise de conscience par la communauté internationale de l'étendue et de la gravité du phénomène de la migration irrégulière et de ses conséquences, et l'urgence et la nécessité d'un cadre normatif institutionnel plus structuré. Cette prise de conscience s'est traduite par l'adoption le 15 novembre 2000 de trois instruments essentiels, mais dont l'efficacité peut être mise en doute dans le cadre de la lutte contre le trafic de migrants : la Convention des Nations Unies contre la criminalité transnationale organisée, le Protocole contre le trafic illicite de migrants par terre, air et mer et enfin le Protocole visant à prévenir, réprimer et punir la traite des personnes, en particulier des femmes et des enfants ${ }^{2}$.

L'intérêt de l'ouvrage d'Anne T. Gallagher et Fiona David, The International Law of Migrant Smuggling, est d'avoir approché le droit international du trafic illicite de migrants non pas en isolation clinique, mais dans le cadre plus général du droit international, fixant ainsi plus solidement la branche de l'immigration irrégulière au tronc plus robuste du droit international coutumier. Ils évitent au passage, le double écueil de l'empirisme stérile ou une description synchronique voire syncrétique des cas de trafic de migrants et du rationalisme abstrait, une analyse purement dogmatique de la normativité sans un effort de confrontation à la pratique étatique. L'ouvrage se propose donc d'appréhender 
le régime juridique international du trafic de migrants à travers deux axes essentiels constitutifs des deux parties du livre. D’une part, une approche plus systémique, une analyse détaillée du cadre normatif général de la migration irrégulière, avec comme prisme d'entrée le droit pénal transnational, le droit de la mer, le droit du contrôle migratoire. Dans ce contexte, les auteurs ont perçu le lien inextricable entre le trafic de migrants et la thématique des droits de l'homme et du droit d'asile, de la responsabilité des États, particulièrement les principes classiques et les tendances nouvelles en matière d'attribution. D'autre part, Anne T. Gallagher et Fiona David abordent, dans la deuxième partie, le trafic de migrants sous un angle plus sectoriel. Ils décrivent la portée de certaines obligations primaires spécifiques notamment l'incrimination du trafic illicite, l'interdiction et le secours en mer, la prévention et la coopération en vue de combattre le trafic, la protection et l'assistance, la détention de migrants victimes du trafic et leur retour.

La structure de l'ouvrage et la démarche des auteurs apparaissent de prime abord assez simples et pertinentes : une présentation du cadre normatif général comprenant les obligations primaires et secondaires des États relatives au trafic illicite de migrants. Les obligations primaires de nature conventionnelle et coutumière découlent notamment du droit international des droits de l'homme, du droit des réfugiés ou des principes de la souveraineté territoriale. Les obligations secondaires sont celles qui gouvernent la responsabilité interna tionale de l'État pour faits internationalement illicites. Il s'agit essentiellement des règles d'attribution et de l'invocation de l'illicéité dans le contexte du trafic de migrants. L'analyse de la due diligence est faite en rapport avec la responsabilité des États donnant ainsi l'impression qu'elle fait partie des règles secondaires de la responsabilité. Or le standard de la due diligence est une espèce particulière de règle primaire qui traduit la normativité en termes de normalité. À ce titre, elle fait partie des principes généraux de droit applicables même en l'absence d'injonction particulière d'une règle.

Ce cadre général est ensuite suivi d'un examen des règles applicables dans des domaines de portée plus spécifique. Mais, comme l'a si bien dit Ludwig Wittgenstein, la complexité des choses réside dans leur facilité apparente. Ainsi, le chapitre 5 qui se veut une analyse de l'obligation spécifique de l'incrimination du trafic illicite de migrants est en réalité une excroissance du chapitre 1 concernant le droit pénal dit transnational. L'examen de l'incrimination se résume alors à une reproduction du cadre normatif fourni par la Convention contre la criminalité transnationale organisée et son Protocole dont l'article 6 prévoit que chaque État Partie adopte les mesures législatives et autres nécessaires pour conférer au trafic illicite de migrants «le caractère d'infraction pénale, lorsque les actes ont été commis intentionnellement et pour en tirer, directement ou indirectement, un avantage financier ou autre avantage matériel». De même, le chapitre 6 n'est autre chose qu'une description plus détaillée de l'article 8 du Protocole. Cet article impose aux États l'obligation d'adopter des mesures contre le trafic illicite de migrants par mer. Or ce même Protocole ainsi que les règles et principes issus du droit de la mer avaient fait l'objet de traitement dans la première partie. De plus, ce qui est considéré comme obligation spécifique de prévention ${ }^{3}$ de coopération ou d'assistance dans le domaine du trafic de migrants est en fait une répétition du cadre normatif général tel que prévu par les articles 10 à 16 du Protocole et de l'obligation de prévention bien établie en droit international coutumier ${ }^{4}$. L'obligation spécifique de la prévention dans le cadre du trafic de migrants inclut toutes les mesures de contrôle des frontières prévues à l'article 11 du Protocole.

Au chapitre 7 de l'ouvrage, les auteurs constatent en filigrane que, malgré le renforcement de la prévention et de la coopération, et l'élargissement des mesures aux frontières, les migrations demeureront largement incontrôlables, à moins que nos États démocratiques ne deviennent des États policiers. Lampleur grandissante des migrations internationales et du trafic de migrants en particulier est une importante conséquence de cette montée des disparités et des inégalités sociales. Dans la mesure où le durcissement des politiques migratoires rend nécessaire l'aide d'une personne pour traverser une frontière internationale, l'efficacité de l'effet dissuasif recherché par les mesures prévues dans la Convention sur la criminalité transnationale organisée et son Protocole ainsi que les mesures draconiennes mises en place par les États, peut être mise en doute. En dépit de la volonté manifeste des États d'incriminer le trafic de migrants et de punir les auteurs de peines plus sévères, l'efficacité de la répression reste inversement proportionnelle à la sévérité du discours politico-judiciaire. On ne peut donc s'empêcher de se poser les interrogations suivantes: contre qui, contre quoi se dirige réellement la lutte contre le trafic illicite de migrants, les trafiquants ou les migrants? Cherche-t-on à réprimer un comportement criminel grave ou n'est-ce qu'une mesure parmi d'autres pour réduire l'immigration irrégulière?

Le Protocole contre le trafic illicite de migrants par terre, air et mer, dispose à son article 18 que: "Chaque État Partie consent à faciliter et à accepter, sans retard injustifié ou déraisonnable, le retour d'une personne qui a été l'objet d'un acte énoncé à l'article 6 du présent Protocole et qui est son ressortissant ou a le droit de résider à titre permanent sur son territoire au moment du retour». Cette disposition pose l'épineuse question du retour des migrants objets
(C) Author(s), 2014. This open-access work is licensed under a Creative Commons Attribution-NonCommercial 4.0 International License.
Cette oeuvre en libre accès fait l'object d'une licence Creative Commons Attribution-NonCommercial 4.0 International. 
de trafic. Il faut reconnaitre avec nos deux auteurs que le droit du retour des migrants objets voire victimes de trafic demeure fluctuant et ses contours imprécis. Si le Protocole reconnait aux États le droit de renvoyer les migrants objets de trafic, l'exercice de ce droit reste néanmoins encadré par les règles fondamentales en matière de protection des droits de l'homme et de la dignité humaine et les principes du droit d'asile, notamment l'obligation de non-refoulement.

The International Law of Migrant Smuggling, malgré ses faiblesses, est à ce jour, une contribution substantielle à la thématique de la migration. S'inscrivant dans une perspective positiviste pour décrire le droit tel qu'il est, Anne T. Gallagher et Fiona David ont, implicitement mis en exergue le paradoxe de la dialectique sein/sollen. L'audace pour nos deux praticiens est d'avoir abordé la question du trafic de migrants à travers le prisme du droit international des droits humains. Ils reconnaissent en fin de compte que les États, dans l'exercice de leur droit souverain à déterminer qui peut entrer et demeurer sur leur territoire, doivent s'acquitter de leur responsabilité et de leur obligation de protéger les droits des migrants. En cherchant à endiguer la migration irrégulière, les États doivent coopérer activement entre eux afin que leurs efforts ne mettent pas en danger les droits humains, notamment le droit des réfugiés à demander l'asile. Le cadre légal et normatif applicable au trafic illicite de migrants doit être mis en œuvre d'une façon plus efficace et sans discrimination afin de respecter les droits humains et les conditions de travail dont chaque migrant doit pouvoir bénéficier. Conformément aux dispositions de ce cadre législatif et normatif, les États et les autres acteurs doivent ainsi aborder les questions migratoires de façon plus conséquente et cohérente. C'est seulement en cela que les opinions publiques prendront la mesure de ce que l'étranger, migrant régulier ou irrégulier, objet ou victime de trafic est un titulaire de droits inaliénables et un justiciable à part entière et que, dans une nouvelle conception de la citoyenneté, sécurité et droits fondamentaux devront bien être réconciliés pour assurer au migrant la protection que requiert sa vulnérabilité. Sir Gerald Fitzmaurice avait certainement raison lorsqu'il affirmait il y a quarante ans déjà: " lawyers must often confront themselves not just with the question of 'what is the law?' but also with the much more challenging one of determining 'what the law is'»5. The International Law of Migrant Smuggling en est la parfaite illustration.

\section{Notes}

1 Article 3 du Protocole contre le trafic illicite de migrants par terre, air et mer.

2 Pour le texte de ces instruments, voir Nations Unies, Recueil des Traités, vol. 2225, p. 209.

3 Anne T. Gallagher, Fiona David, The International Law of Migrant Smuggling, Cambridge, Cambridge University Press, 2014, p. 498 et ss.

4 La nature coutumière de la prévention a été reconnue à plusieurs reprises par la Cour international de Justice. Sur ce point, voir notamment Réserves à la Convention pour la prévention et la répression du crime de génocide, avis consultatif, C.I.J. Recueil 1951, p. 23 ; Application de la Convention pour la prévention et la répression du crime de génocide (Bosnie-Herzégovine c. Serbie-et-Monténégro), arrêt, C.I.J. Recueil 2007, p. 155, par. 432, Usines de pâte à papier sur le fleuve Uruguay (Argentine c. Uruguay), arrêt, C.I.J. Recueil 2010, Opinion individuelle du juge Cançado Trindade, p. 6.

5 G. Fitzmaurice, 'The Future of Public International Law and of the International Legal System in the Circumstances of Today', Special Report, Annuaire IDI, Livre du Centenaire (1973), 251.

Awalou Ouedraogo est Professeur adjoint au Département d'Études internationales au Collège universitaire Glendon, Université York. Ses coordonnées sont : aouedraogo@glendon. yorku.ca.

\title{
Young, Well-Educated and Adaptable: Chilean Exiles in Ontario and Quebec, 1973-2010
}

\author{
$\infty$ \\ Francis Peddie \\ Winnipeg, Manitoba: University of Manitoba Press, 2014
}

$\mathrm{F}$ rancis Peddie explores experiences of exile and adaptation in Young, Well-Educated and Adaptable: Chilean Exiles in Ontario and Quebec, 1973-2010. The central question addressed by Peddie is why so many
Chileans, who remained largely oriented and longed to return to Chile during exile, chose to remain in Canada following the end of the military dictatorship when the prospect of a safe return became a viable option. The author 